\title{
EM-based Bayesian Fusion of Hyperspectral and Multispectral images
}

\author{
Yifan Zhang \\ Northwestern Polytechnical University \\ P. R. China
}

\section{Introduction}

During the last two decades, the number of spectral bands in optical remote sensing technology kept growing steadily going from multispectral (MS) to hyperspectral (HS) data sets. HS images employ hundreds of contiguous spectral bands to capture and process spectral information over a range of wavelenghts, compared to the tens of discrete spectral bands used in MS images (Chang, 2003). This increase in spectral accuracy is delivering more information, allowing a whole range of new and more precise applications. The detailed spectral information of HS images is helpful for interpretation, classification and recognition. However, in remote sensors, usually a trade-off exists between SNR, spatial and spectral resolutions due to physical limitations, data-transfer requirements and some other practical reasons. In most cases, high spatial and spectral resolutions are not available in a single image, which makes the spatial resolution of HS images usually lower than that of MS images (Gomez et al., 2001). In practice, many applications require high accuracy both spectrally and spatially, which inspires research on spatial resolution enhancement techniques for HS image (Gomez et al., 2001; Duijster et al., 2009; Zhang \& He, 2007; Hardie et al., 2004; Eismann \& Hardie, 2005; 2004).

When more than one observation of the scene is available, a popular technique dealing with this limitation is image fusion, a well studied field for more than ten years. As a prototype problem, usually an image of high spectral resolution is combined with an image of high spatial resolution to obtain an image of optimal resolutions both spectrally and spatially. Most fusion techniques for spatial resolution improvement were developed for the specific purpose of enhancing MS image by using a panchromatic (Pan) image of higher spatial resolution, also referred to as pansharpening. Principal component analysis (PCA) (Chavez et al., 1991; Shettigara, 1992) and Intensity-Hue-Saturation (IHS) transform (Carper et al., 1990; Edwards \& Davis, 1994; Tu et al., 2001) based techniques are the most commonly used ones. The Pan image is applied to totally or partially substitute the 1st principal component or intensity component of the coregistered and resampled MS image. To generalize to more than three bands and to reduce spectral degradation, generalized IHS (GIHS) transforms (Tu et al., 2004) and generalized intensity modulation techniques (Alparone et al., 2004) were defined. High-pass filtering and high-pass modulation techniques were developed (Chavez et al., 1991; Shettigara, 1992; Liu \& Moore, 1998), in which spatial high-frequency information is extracted and injected adequately into each band of the MS image. With the rise of multiresolution analysis, many researchers have proposed pansharpening techniques, using Gaussian and Laplacian pyramids as well as discrete decimated and undecimated wavelet transforms (WTs) 
(Aiazzi et al., 2002; Núñez et al., 1999; Shi et al., 2003). A detailed description and comparison of these techniques is given in (Pohl \& Van Genderen, 1998; Wang et al., 2005; Alparone et al., 2007).

In this work, a more general fusion is considered, in which an HS image of low spatial resolution and an MS image of high spatial resolution are observed and fused. Since the high spatial resolution MS image is multiband, the pansharpening techniques cannot be applied directly to the problem of HS and MS image fusion. Usually, a spatial high-frequency component of the MS image is extracted (by PCA, IHS, etc.) first and then injected to the HS image, which may lead to spectral distortion. Techniques using 2D and 3D WTs were also proposed (Gomez et al., 2001; Zhang \& He, 2007), in which the MS and HS images were spectrally and spatially resampled a priori. These two approaches were both capable of improving the spatial resolution of the HS image effectively. However, the performance was highly dependent on the spectral resampling methods adopted. Some researchers proposed statistical estimation techniques for HS and MS image fusion. In (Hardie et al., 2004), MAP estimation based on a spatially varying statistical model is employed to enhance the spatial resolution of HS image. The framework developed was validated for pansharpening but allowed for any number of spectral bands in both the HS and MS images. Extensions of this work applied an extended stochastic mixing model (Eismann \& Hardie, 2005; 2004).

In this work, we treat the problem of fusion of a low-spatial high-spectral resolution observation (HS image) with a high-spatial low-spectral resolution observation (MS image), for the purpose of spatial enhancement of the former. A Bayesian fusion framework is proposed, in which the fusion is accomplished by assuming an observation model for the HS image and a joint statistical model between the HS and MS images. Specifically, an expectation-maximization (EM) algorithm is employed for estimation optimization.

The rest of this work is arranged as follows. In Section 2, mathematical description of the problem concerned is introduced, as well as some related theoretical basis. In Section 3, the EM-based Bayesian fusion framework is elaborated and a practical implementation scheme is provided. In Section 4, simulation experiments with a reference are performed for validation and comparison. Finally, the conclusions are given in Section 5.

\section{Problem description and theoretical basis}

\subsection{Problem description}

The general problem discussed in this paper is to describe a scene $\mathbf{z}$ based on a series of observations, each with specific spatial and spectral resolutions. As a prototype problem, we will consider the case where a low-spatial high-spectral resolution observation $\mathbf{x}$ (HS image) is available together with a high-spatial low-spectral resolution observation y (MS image).

Although the two observations may be presented at different spatial and spectral sampling rates, in this paper, we will assume that all images are equally spatially sampled at a grid of $N$ pixels, sufficiently fine to reveal the spatial resolution of $\mathbf{z}$. Since we will concentrate on the optimization of the spatial resolution and no spectral enhancement will be performed, the spectral sampling rate of $\mathbf{x}$ is sufficient. Each image is presented in band-interleaved-by-pixel lexicographical notation, that is, $\mathbf{z}=\left[\mathbf{z}_{1}^{T}, \mathbf{z}_{2}^{T}, \cdots, \mathbf{z}_{N}^{T}\right]^{T}$ with $\mathbf{z}_{n}=\left[z_{n}^{1}, z_{n}^{2}, \cdots, z_{n}^{P}\right]^{T}$ where $P$ is the number of spectral bands. Similar notations are applied to all related images. Normally, a standard linear observation model is applied for $\mathbf{x}$ :

$$
\mathbf{x}=\mathbf{W z}+\mathbf{n}
$$


where the PSF $\mathbf{W}$ reflects the spatial blurring of the observation $\mathbf{x}$, and $\mathbf{n}$ is the additive Gaussian white noise with covariance $\mathbf{C}_{\mathbf{n}}$.

\subsection{Theoretical basis}

\subsubsection{EM algorithm}

When only the observation $\mathbf{x}$ is available, one way to improve its spatial resolution would be image restoration. A possible treatment of the restoration is splitting it up into a deblurring and a denoising part as was done in (Figueiredo \& Nowak, 2003), where the expectation-maximization (EM) algorithm was employed to solve the problem. In (Duijster et al., 2009), this procedure was extended for multiband images. The key concept in the EM-based restoration procedure is that the observation model for $\mathbf{x}$ is inverted by performing the deblurring and denoising in two separate steps. To accomplish this, the observation model is decomposed as:

$$
\begin{aligned}
& \mathbf{x}=\mathbf{W} \mathbf{s}+\mathbf{n}^{\prime \prime} \\
& \mathbf{s}=\mathbf{z}+\mathbf{n}^{\prime} .
\end{aligned}
$$

In this way, the noise is decomposed into two independent parts $\mathbf{n}^{\prime}$ and $\mathbf{n}^{\prime \prime}$, with $\mathbf{W} \mathbf{n}^{\prime}+\mathbf{n}^{\prime \prime}=$ $\mathbf{n}$. The spatial-invariance of $\mathbf{W}$ guarantees a semi positive-definite covariance for $\mathbf{n}^{\prime \prime}$. If $\mathbf{W}$ would be not translation-invariant, a rescaling is required (see (Figueiredo \& Nowak, 2003)). The splitting up leaves the option to divide the originally assumed Gaussian white noise $\mathbf{n}$ into two parts. Choosing $\mathbf{n}^{\prime}$ to be white with $p\left(\mathbf{n}^{\prime}\right)=\phi\left(0, \mathbf{C}_{\mathbf{n}}\right)$ facilitates the denoising problem (3). However, $\mathbf{W}$ colors the noise so that $\mathbf{n}^{\prime \prime}$ becomes colored with $p\left(\mathbf{n}^{\prime \prime}\right)=\phi\left(0, \mathbf{C}_{\mathbf{n}}-\mathbf{W C}_{\mathbf{n}} \mathbf{W}^{T}\right)$. When the largest part of the original noise appears into $\mathbf{n}^{\prime}, \mathbf{n}^{\prime \prime}$ can be neglected, making (2) a pure deblurring problem.

The estimation problem concerned can be then described as:

$$
\begin{aligned}
\hat{\mathbf{z}} & =\arg \max _{\mathbf{z}} p(\mathbf{z} \mid \mathbf{x}, \mathbf{s}) \\
& =\arg \max _{\mathbf{z}} p(\mathbf{x}, \mathbf{s} \mid \mathbf{z}) p(\mathbf{z})
\end{aligned}
$$

which is solved using the iterative EM algorithm. At each iteration $k$, the EM algorithm involves two steps:

- The E-step computes the conditional expectation of the complete log-likelihood, the so-called $Q$-function, given the observation $\mathbf{x}$ and an estimate of $\mathbf{z}$ acquired in the previous iteration:

$$
Q\left(\mathbf{z}, \hat{\mathbf{z}}^{(k-1)}\right)=\mathbb{E}\left[\log (p(\mathbf{x}, \mathbf{s} \mid \mathbf{z}) p(\mathbf{z})) \mid \mathbf{x}, \hat{\mathbf{z}}^{(k-1)}\right] .
$$

- The M-step maximizes the $Q$-function and updates the estimate of $\mathbf{z}$ :

$$
\hat{\mathbf{z}}^{(k)}=\arg \max _{\mathbf{z}} Q\left(\mathbf{z}, \hat{\mathbf{z}}^{(k-1)}\right) .
$$

\subsubsection{E-step}

Conditioned on $\mathbf{s}, \mathbf{x}$ is independent of $\mathbf{z}$ (see (2)), therefore:

$$
\begin{aligned}
p(\mathbf{x}, \mathbf{s} \mid \mathbf{z}) p(\mathbf{z}) & =p(\mathbf{x} \mid \mathbf{s}, \mathbf{z}) p(\mathbf{s} \mid \mathbf{z}) p(\mathbf{z}) \\
& =p(\mathbf{x} \mid \mathbf{s}) p(\mathbf{s} \mid \mathbf{z}) p(\mathbf{z}) \\
& \propto p(\mathbf{s} \mid \mathbf{z}) p(\mathbf{z}) .
\end{aligned}
$$


From Equation (3), one has $p(\mathbf{s} \mid \mathbf{z})=\phi\left(\mathbf{z}, \mathbf{C}_{\mathbf{n}}\right)$. When a Gaussian prior is assumed for $\mathbf{z}$, one has

$$
p(\mathbf{z})=\phi\left(\boldsymbol{\mu}_{\mathbf{z}}, \mathbf{C}_{\mathbf{z}}\right)
$$

with

$$
\begin{aligned}
& \boldsymbol{\mu}_{\mathbf{z}}=\mathbb{E}[\mathbf{z}] \\
& \mathbf{C}_{\mathbf{z}}=\mathbb{E}\left[(\mathbf{z}-\mathbb{E}[\mathbf{z}])(\mathbf{z}-\mathbb{E}[\mathbf{z}])^{T}\right]
\end{aligned}
$$

which can be estimated from an estimate of $\mathbf{z}$ obtained in the previous iteration $\left(\hat{\mathbf{z}}^{(k-1)}\right)$. Hence, the complete log-likelihood can be expressed as:

$$
\log (p(\mathbf{x}, \mathbf{s} \mid \mathbf{z}) p(\mathbf{x} \mid \mathbf{y})) \propto-\frac{1}{2}(\mathbf{z}-\mathbf{s})^{T} \mathbf{C}_{\mathbf{n}}^{-1}(\mathbf{z}-\mathbf{s})-\frac{1}{2}\left(\mathbf{z}-\boldsymbol{\mu}_{\mathbf{z}}\right)^{T} \mathbf{C}_{\mathbf{z}}^{-1}\left(\mathbf{z}-\boldsymbol{\mu}_{\mathbf{z}}\right) .
$$

Since the $\mathbf{z}$-dependent part of this expression is linear in $\mathbf{s}$, finding the $Q$-function or the expectation of (10) comes down to finding the expectation of $\mathbf{s}$, conditioned on the observation $\mathbf{x}$ and an estimate of $\mathbf{z}$ from last iteration. Denoting this expectation as $\hat{\mathbf{s}}^{(k)}$, the final expression for the $Q$-function becomes:

$$
Q\left(\mathbf{z}, \hat{\mathbf{z}}^{(k-1)}\right) \propto-\frac{1}{2}\left(\mathbf{z}-\hat{\mathbf{s}}^{(k)}\right)^{T} \mathbf{C}_{\mathbf{n}}^{-1}\left(\mathbf{z}-\hat{\mathbf{s}}^{(k)}\right)-\frac{1}{2}\left(\mathbf{z}-\boldsymbol{\mu}_{\mathbf{z}}\right)^{T} \mathbf{C}_{\mathbf{z}}^{-1}\left(\mathbf{z}-\boldsymbol{\mu}_{\mathbf{z}}\right) .
$$

\subsubsection{Calculation of $\hat{\mathbf{s}}^{(k)}$}

$\hat{\mathbf{s}}^{(k)}$ can be obtained from the conditional pdf of $\mathbf{s}$ given the observation $\mathbf{x}$ and an estimate of $\mathbf{z}$ from the previous iteration:

$$
\begin{aligned}
p\left(\mathbf{s} \mid \mathbf{x}, \hat{\mathbf{z}}^{(k-1)}\right) & =\frac{p\left(\mathbf{x} \mid \mathbf{s}, \hat{\mathbf{z}}^{(k-1)}\right) p\left(\mathbf{s} \mid \hat{\mathbf{z}}^{(k-1)}\right)}{p\left(\mathbf{x} \mid \hat{\mathbf{z}}^{(k-1)}\right)} \\
& =\frac{p(\mathbf{x} \mid \mathbf{s}) p\left(\mathbf{s} \mid \hat{\mathbf{z}}^{(k-1)}\right)}{p\left(\mathbf{x} \mid \hat{\mathbf{z}}^{(k-1)}\right)} \\
& \propto p(\mathbf{x} \mid \mathbf{s}) p\left(\mathbf{s} \mid \hat{\mathbf{z}}^{(k-1)}\right)
\end{aligned}
$$

where the first pdf comes from (2) and the second from (3)

$$
\begin{aligned}
p(\mathbf{x} \mid \mathbf{s}) & =\phi\left(\mathbf{W s}, \mathbf{C}_{\mathbf{n}}-\mathbf{W} \mathbf{C}_{\mathbf{n}} \mathbf{W}^{T}\right) \\
p\left(\mathbf{s} \mid \hat{\mathbf{z}}^{(k-1)}\right) & =\phi\left(\hat{\mathbf{z}}^{(k-1)}, \mathbf{C}_{\mathbf{n}}\right) .
\end{aligned}
$$

As a result, the conditional expectation of $\mathbf{s}$ can then be obtained as:

$$
\begin{aligned}
\hat{\mathbf{s}}^{(k)} & =\mathbb{E}\left[\mathbf{s} \mid \mathbf{x}, \mathbf{z}^{(k-1)}\right] \\
& =\int \mathbf{s} p(\mathbf{x} \mid \mathbf{s}) p\left(\mathbf{s} \mid \hat{\mathbf{z}}^{(k-1)}\right) d \mathbf{s} \\
& =\hat{\mathbf{z}}^{(k-1)}+\mathbf{W}^{T}\left(\mathbf{x}-\mathbf{W} \hat{\mathbf{z}}^{(k-1)}\right) .
\end{aligned}
$$




\subsubsection{M-step}

In this step, the estimate of $\mathbf{z}$ is updated by maximizing the $Q$-function obtained in (11):

$$
\begin{aligned}
\hat{\mathbf{z}}^{(k)} & =\arg \max _{\mathbf{z}} Q\left(\mathbf{z}, \hat{\mathbf{z}}^{(k-1)}\right) \\
& =\mathbf{C}_{\hat{\mathbf{z}}^{(k-1)}}\left(\mathbf{C}_{\hat{\mathbf{z}}^{(k-1)}}+\mathbf{C}_{\mathbf{n}}\right)^{-1} \hat{\mathbf{s}}^{(k)}+\mathbf{C}_{\mathbf{n}}\left(\mathbf{C}_{\hat{\mathbf{z}}^{(k-1)}}+\mathbf{C}_{\mathbf{n}}\right)^{-1} \boldsymbol{\mu}_{\hat{\mathbf{z}}^{(k-1)}} .
\end{aligned}
$$

Since no auxiliary information is used in the whole procedure, its performance in spatial enhancement is usually quite limited.

\subsubsection{Bayesian fusion using MAP estimation}

When the high-spatial low-spectral resolution observation $\mathbf{y}$ is also available, image fusion technique would be a solution for spatial enhancement of $\mathbf{x}$. In a Bayesian framework, an estimate of $\mathbf{z}$ can be obtained from the conditional pdf given both observations using MAP estimation (Hardie et al., 2004):

$$
\begin{aligned}
\hat{\mathbf{z}} & =\arg \max _{\mathbf{z}} p(\mathbf{z} \mid \mathbf{x}, \mathbf{y}) \\
& =\arg \max _{\mathbf{z}} p(\mathbf{x} \mid \mathbf{z}) p(\mathbf{z} \mid \mathbf{y}) .
\end{aligned}
$$

The first pdf is obtained from the observation model for $\mathbf{x}$ (see (1)):

$$
p(\mathbf{x} \mid \mathbf{z})=\phi\left(\mathbf{W z}, \mathbf{C}_{\mathbf{n}}\right) .
$$

By assuming a jointly Gaussian distribution between $\mathbf{z}$ and $\mathbf{y}$, the conditional pdf $p(\mathbf{z} \mid \mathbf{y})$ would also be a Gaussian (Hardie et al., 2004):

$$
p(\mathbf{z} \mid \mathbf{y})=\phi\left(\boldsymbol{\mu}_{\mathbf{z} \mid \mathbf{y}}, \mathbf{C}_{\mathbf{z} \mid \mathbf{y}}\right)
$$

with

$$
\begin{aligned}
& \boldsymbol{\mu}_{\mathbf{z} \mid \mathbf{y}}=\mathbb{E}[\mathbf{z}]+\mathbf{C}_{\mathbf{z}, \mathbf{y}} \mathbf{C}_{\mathbf{y}}^{-1}(\mathbf{y}-\mathbb{E}[\mathbf{y}]) \\
& \mathbf{C}_{\mathbf{z} \mid \mathbf{y}}=\mathbf{C}_{\mathbf{z}}-\mathbf{C}_{\mathbf{z}, \mathbf{y}} \mathbf{C}_{\mathbf{y}}^{-1} \mathbf{C}_{\mathbf{z}, \mathbf{y}}^{T}
\end{aligned}
$$

where

$$
\mathbf{C}_{\mathbf{z}, \mathbf{y}}=\mathbb{E}\left[(\mathbf{z}-\mathbb{E}[\mathbf{z}])(\mathbf{y}-\mathbb{E}[\mathbf{y}])^{T}\right] .
$$

After some calculation, the following solution can be easily obtained:

$$
\hat{\mathbf{z}}=\boldsymbol{\mu}_{\mathbf{z} \mid \mathbf{y}}+\mathbf{C}_{\mathbf{z} \mid \mathbf{y}} \mathbf{W}^{T}\left(\mathbf{W C}_{\mathbf{z} \mid \mathbf{y}} \mathbf{W}^{T}+\mathbf{C}_{\mathbf{n}}\right)^{-1}\left(\mathbf{y}-\mathbf{W} \boldsymbol{\mu}_{\mathbf{z} \mid \mathbf{y}}\right) .
$$

In this fusion approach, ideally, the fused result $\hat{\mathbf{z}}$ has the spectral resolution of $\mathbf{x}$ and the spatial resolution of $\mathbf{y}$. As a result, the spatial resolution of $\hat{\mathbf{z}}$ is limited to that of $\mathbf{y}$.

\section{Bayesian fusion based on EM algorithm}

In this section, a new Bayesian fusion approach for HS and MS images is proposed, which employs both the EM algorithm presented in Section 2.2.1 and Bayesian fusion framework explained in Section 2.2.2, for the purpose of performance improvement. Based on the 
splitting-up strategy ((2) and (3)), the objective of the fusion problem discussed is to find an estimate of $\mathbf{z}$ by:

$$
\begin{aligned}
\hat{\mathbf{z}} & =\arg \max _{\mathbf{z}} p(\mathbf{z} \mid \mathbf{x}, \mathbf{y}, \mathbf{s}) \\
& =\arg \max _{\mathbf{z}} p(\mathbf{x}, \mathbf{s} \mid \mathbf{z}) p(\mathbf{z} \mid \mathbf{y}) .
\end{aligned}
$$

Employing the EM algorithm, the proposed fusion approach is an iterative procedure with two major steps in each iteration $k$ :

- The E-step computes the conditional expectation of the complete log-likelihood, the so-called $Q$-function, given both observations ( $\mathbf{x}$ and $\mathbf{y}$ ) and an estimate of $\mathbf{z}$ acquired in the previous iteration:

$$
Q\left(\mathbf{z}, \hat{\mathbf{z}}^{(k-1)}\right)=\mathbb{E}\left[\log (p(\mathbf{x}, \mathbf{s} \mid \mathbf{z}) p(\mathbf{z} \mid \mathbf{y})) \mid \mathbf{x}, \mathbf{y}, \hat{\mathbf{z}}^{(k-1)}\right] .
$$

- The M-step maximizes the $Q$-function and updates the estimate of $\mathbf{z}$ :

$$
\hat{\mathbf{z}}^{(k)}=\arg \max _{\mathbf{z}} Q\left(\mathbf{z}, \hat{\mathbf{z}}^{(k-1)}\right) .
$$

\subsection{E-step}

Since conditioned on $\mathbf{s}, \mathbf{x}$ is independent of $\mathbf{z}$ (see (2)), the following can be obtained:

$$
\begin{aligned}
p(\mathbf{x}, \mathbf{s} \mid \mathbf{z}) p(\mathbf{z} \mid \mathbf{y}) & =p(\mathbf{x} \mid \mathbf{s}, \mathbf{z}) p(\mathbf{s} \mid \mathbf{z}) p(\mathbf{z} \mid \mathbf{y}) \\
& =p(\mathbf{x} \mid \mathbf{s}) p(\mathbf{s} \mid \mathbf{z}) p(\mathbf{z} \mid \mathbf{y}) \\
& \propto p(\mathbf{s} \mid \mathbf{z}) p(\mathbf{z} \mid \mathbf{y}) .
\end{aligned}
$$

From (3), one has $p(\mathbf{s} \mid \mathbf{z})=\phi\left(\mathbf{z}, \mathbf{C}_{\mathbf{n}}\right)$. As for $p(\mathbf{z} \mid \mathbf{y})$, we will assume that $\mathbf{z}$ and $\mathbf{y}$ are jointly normally distributed as in (Hardie et al., 2004), so that the conditional distribution is also a normal (see (19)). As a result,

$$
\log (p(\mathbf{x}, \mathbf{s} \mid \mathbf{z}) p(\mathbf{z} \mid \mathbf{y})) \propto-\frac{1}{2}(\mathbf{z}-\mathbf{s})^{T} \mathbf{C}_{\mathbf{n}}^{-1}(\mathbf{z}-\mathbf{s})-\frac{1}{2}\left(\mathbf{z}-\boldsymbol{\mu}_{\mathbf{z} \mid \mathbf{y}}\right)^{T} \mathbf{C}_{\mathbf{z} \mid \mathbf{y}}^{-1}\left(\mathbf{z}-\boldsymbol{\mu}_{\mathbf{z} \mid \mathbf{y}}\right) .
$$

Since the z-dependent part of this expression is linear in $\mathbf{s}$, finding the $Q$-function or the expectation of (26) comes down to finding the expectation of $\mathbf{s}$, conditioned on both observations ( $\mathbf{x}$ and $\mathbf{y}$ ) as well as $\hat{\mathbf{z}}^{(k-1)}$. We will denote this expectation as $\hat{\mathbf{s}}^{(k)}$, so that the final expression for the $Q$-function becomes:

$$
Q\left(\mathbf{z}, \hat{\mathbf{z}}^{(k-1)}\right) \propto-\frac{1}{2}\left(\mathbf{z}-\hat{\mathbf{s}}^{(k)}\right)^{T} \mathbf{C}_{\mathbf{n}}^{-1}\left(\mathbf{z}-\hat{\mathbf{s}}^{(k)}\right)-\frac{1}{2}\left(\mathbf{z}-\boldsymbol{\mu}_{\mathbf{z} \mid \mathbf{y}}\right)^{T} \mathbf{C}_{\mathbf{z} \mid \mathbf{y}}^{-1}\left(\mathbf{z}-\boldsymbol{\mu}_{\mathbf{z} \mid \mathbf{y}}\right) .
$$

\subsection{Calculation of $\hat{\mathbf{s}}^{(k)}$}

The pdf of $\mathbf{s}$ given both observations and an estimate of $\mathbf{z}$ from the previous iteration is described as following:

$$
\begin{aligned}
p\left(\mathbf{s} \mid \mathbf{x}, \mathbf{y}, \hat{\mathbf{z}}^{(k-1)}\right) & =\frac{p\left(\mathbf{x} \mid \mathbf{s}, \mathbf{y}, \hat{\mathbf{z}}^{(k-1)}\right)}{p\left(\mathbf{x} \mid \mathbf{y}, \hat{\mathbf{z}}^{(\mathbf{k}-1)}\right)} \cdot p\left(\mathbf{s} \mid \mathbf{y}, \hat{\mathbf{z}}^{(k-1)}\right) \\
& =\frac{p\left(\mathbf{x} \mid \mathbf{s}, \mathbf{y}, \hat{\mathbf{z}}^{(k-1)}\right)}{p\left(\mathbf{x} \mid \mathbf{y}, \hat{\mathbf{z}}^{(k-1)}\right)} \cdot \frac{p\left(\hat{\mathbf{z}}^{(k-1)} \mid \mathbf{s}, \mathbf{y}\right) p(\mathbf{s} \mid \mathbf{y})}{p\left(\hat{\mathbf{z}}^{(k-1)} \mid \mathbf{y}\right)} .
\end{aligned}
$$


Since conditioned on $\mathbf{s}, \mathbf{x}$ is independent of $\hat{\mathbf{z}}^{(k-1)}\left(\right.$ see (2)), $\hat{\mathbf{z}}^{(k-1)}$ is independent of $\mathbf{y}$ (see (3)), besides $\mathbf{x}$ and $\mathbf{y}$ are independent, the conditional pdf can then be rewritten as:

$$
\begin{aligned}
p\left(\mathbf{s} \mid \mathbf{x}, \mathbf{y}, \hat{\mathbf{z}}^{(k-1)}\right) & =\frac{p(\mathbf{x} \mid \mathbf{s}) p\left(\hat{\mathbf{z}}^{(k-1)} \mid \mathbf{s}\right) p(\mathbf{s} \mid \mathbf{y})}{p\left(\mathbf{x} \mid \hat{\mathbf{z}}^{(k-1)}\right) p\left(\hat{\mathbf{z}}^{(k-1)} \mid \mathbf{y}\right)} \\
& \propto p(\mathbf{x} \mid \mathbf{s}) p\left(\hat{\mathbf{z}}^{(k-1)} \mid \mathbf{s}\right) p(\mathbf{s} \mid \mathbf{y})
\end{aligned}
$$

with

$$
\begin{aligned}
p(\mathbf{x} \mid \mathbf{s}) & =\phi\left(\mathbf{W} \mathbf{s}, \mathbf{C}_{\mathbf{n}}-\mathbf{W C}_{\mathbf{n}} \mathbf{W}^{T}\right) \\
p\left(\hat{\mathbf{z}}^{(k-1)} \mid \mathbf{s}\right) & =\phi\left(\mathbf{s}, \mathbf{C}_{\mathbf{n}}\right) \\
p(\mathbf{s} \mid \mathbf{y}) & =\phi\left(\boldsymbol{\mu}_{\hat{\mathbf{z}}^{(k-1)} \mid \mathbf{y}^{\prime}} \mathbf{C}_{\hat{\mathbf{z}}^{(k-1)} \mid \mathbf{y}}+\mathbf{C}_{\mathbf{n}}\right)
\end{aligned}
$$

where the first expression is derived from (2), the second from (3) and the third from the prior model assumption combined with (3). Thus, an estimate of the expectation of $\mathbf{s}$ leads to:

$$
\begin{aligned}
\hat{\mathbf{s}}^{(k)} & =\mathbb{E}\left[\mathbf{s} \mid \mathbf{x}, \mathbf{y}, \mathbf{z}^{(k-1)}\right] \\
& =\int \mathbf{s} p(\mathbf{x} \mid \mathbf{s}) p\left(\hat{\mathbf{z}}^{(k-1)} \mid \mathbf{s}\right) p(\mathbf{s} \mid \mathbf{y}) d \mathbf{s} \\
& =\boldsymbol{\mu}+\mathbf{C}(\mathbf{x}-\mathbf{W} \boldsymbol{\mu})
\end{aligned}
$$

with

$$
\begin{aligned}
\boldsymbol{\mu} & =\mathbf{B}\left[\mathbf{C}_{\mathbf{n}}^{-1} \hat{\mathbf{z}}^{(k-1)}+\left(\mathbf{C}_{\hat{\mathbf{z}}^{(k-1)} \mid \mathbf{y}}+\mathbf{C}_{\mathbf{n}}\right)^{-1} \boldsymbol{\mu}_{\hat{\mathbf{z}}^{(k-1)} \mid \mathbf{y}}\right] . \\
\mathbf{C} & =\mathbf{B} \mathbf{W}^{T}\left[\mathbf{C}_{\mathbf{n}}+\mathbf{W}\left(\mathbf{B}-\mathbf{C}_{\mathbf{n}}\right) \mathbf{W}^{T}\right]^{-1} \\
\mathbf{B} & =\left[\mathbf{C}_{\mathbf{n}}^{-1}+\left(\mathbf{C}_{\hat{\mathbf{z}}^{(k-1)} \mid \mathbf{y}}+\mathbf{C}_{\mathbf{n}}\right)^{-1}\right]^{-1}
\end{aligned}
$$

\subsection{M-step}

In this step, the estimate of $\mathbf{z}$ is updated by maximizing the $Q$-function in (27), which leads to:

$$
\begin{aligned}
\hat{\mathbf{z}}^{(k)} & =\arg \max _{\mathbf{z}} Q\left(\mathbf{z}, \hat{\mathbf{z}}^{(k-1)}\right) \\
& =\mathbf{C}_{\hat{\mathbf{z}}^{(k-1)} \mid \mathbf{y}}\left(\mathbf{C}_{\hat{\mathbf{z}}^{(k-1)} \mid \mathbf{y}}+\mathbf{C}_{\mathbf{n}}\right)^{-1} \hat{\mathbf{s}}^{(k)}+\mathbf{C}_{\mathbf{n}}\left(\mathbf{C}_{\hat{\mathbf{z}}^{(k-1)} \mid \mathbf{y}}+\mathbf{C}_{\mathbf{n}}\right)^{-1} \boldsymbol{\mu}_{\hat{\mathbf{z}}^{(k-1)} \mid \mathbf{y}} .
\end{aligned}
$$

\subsection{Discussion}

Remark that the obtained expression in the E-step (28) is a combination of the expressions obtained by a restoration of $\mathbf{x}$ and a fusion of $\mathbf{x}$ with $\mathbf{y}$. Indeed, when no high-spatial resolution image $(\mathbf{y})$ is available, (28) would reduce to (15) which is a deconvolution result of $\mathbf{x}$. On the other hand, if no EM algorithm would be applied, the MAP estimation of (22) would lead to (21) which is a Bayesian fusion of $\mathbf{x}$ and $\mathbf{y}$ using MAP estimation. The obtained expression in the M-step (29) makes use of both observations. Without the use of $\mathbf{y}$, the expression would reduce to (16), accounting for the interband correlation of $\hat{\mathbf{z}}^{(\mathbf{k}-\mathbf{1})}$. While in (29), the correlation between $\hat{\mathbf{z}}^{(\mathbf{k}-\mathbf{1})}$ and $\mathbf{y}$ are also accounted for. Therefore, the proposed approach is actually a combination of EM-based restoration and Bayesian fusion. 


\section{Experiments and analysis}

\subsection{Implementation}

\subsubsection{Noise covariance}

The noise covariance $\mathbf{C}_{\mathbf{n}}$ is required in the estimation. In this paper, instead of assuming it is known, it is estimated from $\hat{\mathbf{z}}^{(k-1)}$. The noise is assumed to be spectrally uncorrelated, so that $\mathrm{C}_{\mathbf{n}}$ is diagonal:

$$
\mathbf{C}_{\mathbf{n}}=\left[\begin{array}{cccc}
\mathbf{C}_{\mathbf{n}_{1}} & \mathbf{0} & \cdots & \mathbf{0} \\
\mathbf{0} & \mathbf{C}_{\mathbf{n}_{2}} & \cdots & \mathbf{0} \\
\vdots & \vdots & \ddots & \vdots \\
\mathbf{0} & \mathbf{0} & \cdots & \mathbf{C}_{\mathbf{n}_{N}}
\end{array}\right] \text { with } \mathbf{C}_{\mathbf{n}_{n}}=\left[\begin{array}{cccc}
\hat{\sigma}_{1}^{2} & 0 & \cdots & 0 \\
0 & \hat{\sigma}_{2}^{2} & \cdots & 0 \\
\vdots & \vdots & \ddots & \vdots \\
0 & 0 & \cdots & \hat{\sigma}_{P}^{2}
\end{array}\right] \quad(n=1,2, \cdots, N)
$$

The diagonal elements in the noise covariance can be estimated in several ways. In this work, we employ the well-known estimator by Donoho (Donoho \& Johnstone, 1995):

$$
\hat{\sigma}_{p}=\frac{\operatorname{median}\left(\left|\hat{\mathbf{z}}_{p}^{(1, \text { diag })}\right|\right)}{0.6745}
$$

where $\hat{\mathbf{z}}_{p}^{(1, \text { diag })}$ represents the wavelet diagonal subband at the first resolution scale of the $p$ th $(p=1,2, \cdots, P)$ spectral band of $\hat{\mathbf{z}}^{(k-1)}$.

\subsubsection{Spatial independence}

Estimating the full size covariance matrix $\mathbf{C}_{\mathbf{z} \mid \mathbf{y}}$ (of size $N P \times N P$ ) is impractical for a typical size HS image. To keep the calculations feasible, we follow a similar strategy as employed in (Hardie et al., 2004). The pixels in $\mathbf{z}$ are assumed to be spatially conditionally independent, so that the conditional expectation and covariance can be estimated independently for each individual pixel:

$$
\begin{aligned}
\boldsymbol{\mu}_{\mathbf{z} \mid \mathbf{y}}= & {\left[\boldsymbol{\mu}_{\mathbf{z}_{1} \mid \mathbf{y}_{1},}^{T} \boldsymbol{\mu}_{\mathbf{z}_{2} \mid \mathbf{y}_{2}}^{T}, \cdots, \boldsymbol{\mu}_{\mathbf{z}_{N} \mid \mathbf{y}_{N}}^{T}\right]^{T} } \\
\mathbf{C}_{\mathbf{z} \mid \mathbf{y}}= & {\left[\begin{array}{cccc}
\mathbf{C}_{\mathbf{z}_{1} \mid \mathbf{y}_{1}} & \mathbf{0} & \cdots & \mathbf{0} \\
\mathbf{0} & \mathbf{C}_{\mathbf{z}_{2} \mid \mathbf{y}_{2}} & \cdots & \mathbf{0} \\
\vdots & \vdots & \ddots & \vdots \\
\mathbf{0} & \mathbf{0} & \cdots & \mathbf{C}_{\mathbf{z}_{N} \mid \mathbf{y}_{N}}
\end{array}\right] }
\end{aligned}
$$

where the individual conditional expectation and covariance is estimated as:

$$
\begin{aligned}
& \boldsymbol{\mu}_{\mathbf{z}_{n} \mid \mathbf{y}_{n}}=\mathbb{E}\left[\mathbf{z}_{n}\right]+\mathbf{C}_{\mathbf{z}_{n}, \mathbf{y}_{n}} \mathbf{C}_{\mathbf{y}_{n}}^{-1}\left(\mathbf{y}_{n}-\mathbb{E}\left[\mathbf{y}_{n}\right]\right) \\
& \mathbf{C}_{\mathbf{z}_{n} \mid \mathbf{y}_{n}}=\mathbf{C}_{\mathbf{z}_{n}}-\mathbf{C}_{\mathbf{z}_{n}, \mathbf{y}_{n}} \mathbf{C}_{\mathbf{y}_{n}}^{-1} \mathbf{C}_{\mathbf{z}_{n}, \mathbf{y}_{n}}^{T} .
\end{aligned}
$$

In this work, all related expectation as well as self- and cross-covariances are globally estimated, which denotes that they are constants for each pixel.

\subsubsection{Block-by-block estimation strategy}

Since $\mathbf{C}_{\mathbf{n}}$ is diagonal and $\mathbf{C}_{\mathbf{z} \mid \mathbf{y}}$ is block-diagonal, $\boldsymbol{\mu}=\left[\boldsymbol{\mu}_{1}^{T}, \boldsymbol{\mu}_{2}^{T}, \cdots, \boldsymbol{\mu}_{N}^{T}\right]^{T}$ and $\mathbf{B}$ is also block-diagonal. However, because of the effect of $\mathbf{W}$ and matrix inversion, $\mathbf{C}$ is not 
block-diagonal. The calculation of $\mathbf{C}$ and thus the estimation in (28) cannot be implemented pixel by pixel. The matrix with size $N P \times N P$ is obviously too large to be practical. To solve this problem, we design a practical implementation scheme, in which $\mathbf{C}$ and thus $\hat{\mathbf{s}}^{(k)}$ are calculated block by block. The image is divided into non-overlapping blocks with an appropriate size (with $M$ pixels, in this paper, a $16 \times 16$ square is used), which is sufficiently large for the PSF simulation and sufficiently small for keeping the calculations feasible. In this work, $\mathbf{W}$ models a space-invariant periodic convolution in the image domain. $\mathbf{W}$ is then a square block-circulant matrix $($ size $N P \times N P$ ) constructed from the convolution kernel. Using the same convolution kernel, we can construct $\mathbf{W}_{b}$ (size $M P \times M P$ ) in the same manner, which performs the blurring on each block in $\mathbf{z}$, mimicking the way that $\mathbf{W}$ performs on $\mathbf{z}$. The calculation of $\mathbf{C}$ and the estimation of $\mathbf{s}$ are then implemented block by block, using $\mathbf{W}_{b}$ instead of $\mathbf{W}$.

\subsection{Experimental setup}

In this work, simulation experiments with a reference are employed for performance validation and comparison, so that the fused results can be compared to the reference. Performances of fusion techniques are usually difficult to be measured only based on observation, especially for multiband images. Objective and quantitative analysis can contribute to a more comprehensive evaluation. In this work, we employ the SNR in decibels between the result and the reference as the performance evaluation index:

$$
\operatorname{SNR}(\mathbf{Z}, \hat{\mathbf{Z}})=10 \log _{10} \frac{\sum \mathbf{Z}^{2}}{\sum(\mathbf{Z}-\hat{\mathbf{Z}})^{2}} .
$$

For the first set of experiments (Test 1), an AVIRIS HS image of NW Indiana's Indian Pine test site, USA in 1992 with 220 bands is employed. To construct the experimental data, we select 60 continuous bands (bands 11-70) and $128 \times 128$ pixels in each band, avoiding atmospheric water bands and bands with low SNR. To limit processing time, a 10-band HS reference image is constructed by averaging over 6 adjacent bands successively. It is then spatially smoothed by a Gaussian low-pass filter with a standard deviation of 1.2 and Gaussian noise is also added to acquire $\mathbf{x}$. A 3-band image $\mathbf{y}$ is obtained by averaging the original 60-band image over 20 adjacent bands successively.

In the second set of experiments (Test 2), we apply the presented framework to a specific case of fusion, pansharpening, where an MS image of low spatial resolution is fused with a Pan image of high spatial resolution. For this, a set of color-composite Landsat images ( 3 bands, $30 \mathrm{~m}$ resolution) and a SPOT Pan image (10m resolution) covering an area near London are used as test data. To be able to use the original Landsat image as a reference, we smooth it with a Gaussian low-pass filter with a standard deviation of 1.2 and Gaussian noise is also added to obtain $\mathbf{x}$. A degraded SPOT Pan image to $30 \mathrm{~m}$ is used as $\mathbf{y}$.

For initialization of EM algorithm, we set $\hat{\mathbf{z}}^{(0)}=\mathbf{x}$.

\subsection{Experimental results and analysis}

\subsubsection{Algorithm convergence}

In this part, experiments are performed to validate the proposed fusion framework. In Test 1 , general HS and MS image fusion is discussed, using $\mathbf{x}$ with noise level of $25 \mathrm{~dB}$. In Test 2, the proposed approach is validated for the specific case of pansharpening, using $\mathbf{x}$ with noise level of $20 \mathrm{~dB}$. In Fig. 1, the SNRs between the reference and fused images as a function of 


\begin{tabular}{cccccc}
\hline Noise level & 40 & 35 & 30 & 25 & 20 \\
\hline Test 1 & 35.4405 & 35.3011 & 34.7885 & 33.5456 & 29.0465 \\
Test 2 & 25.3240 & 25.1845 & 24.9342 & 24.6979 & 24.2355 \\
\hline
\end{tabular}

Table 1. SNR in fusion tests with different noise levels

the number of iterations of the EM algorithm involved are shown. It can be observed that the SNR increases sharply in the first several iterations and converges after around 10 iterations. The proposed approach is also validated for $\mathbf{x}$ with different noise levels (20-40dB) in both Test 1 and 2, and comparable results are observed. Fig. 2 shows $\mathbf{x}$ with specific noise levels and the corresponding fused results, as well as the reference from Test 2 . The slight differences among all the fused images illustrate the excellent noise-resistance of the proposed fusion approach. The SNRs between the reference and fused images produced in different experiments are listed in Table 1, together with the original noise levels of $\mathbf{x}$.

\subsubsection{Knowledge about $\mathrm{W}$}

In the estimation of (28), the PSF $\mathbf{W}$ is required. Nevertheless, knowledge about the PSF is usually partly or totally unknown in practice. In this part, we will discuss the influence of (lack of) knowledge of $\mathbf{W}$ on the fused result. To address this problem, we arrange the following experiment. We employ the experimental data constructed in Section 4.2, while using Gaussian low-pass filters with standard deviation $\sigma_{1}$ to model $\mathbf{W}$, with $\sigma_{1} \in[0.3,2.1]$ with a step of 0.1 (the actual $\sigma_{1}=1.2$ ). The SNRs between the fused and reference images as a function of $\sigma_{1}$ are shown in Fig. 3. It can be observed that a little underestimated or overestimated $\mathbf{W}\left(\sigma_{1} \in[1.0,1.4]\right)$ can still produce fairly good fused results. It seems that an overestimated $\mathbf{W}$ has even less influence on the fusion results than an underestimated $\mathbf{W}$,

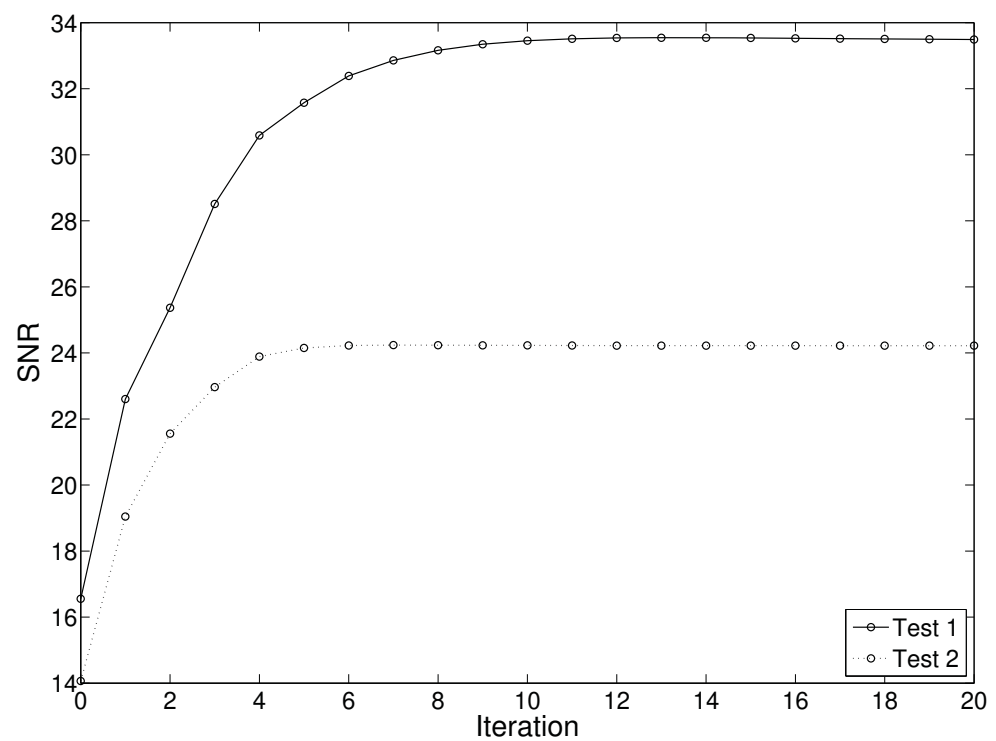

Fig. 1. SNR in function of number of iterations. 


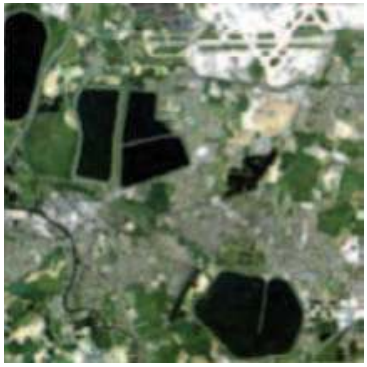

(a) $\mathbf{x}(40 \mathrm{~dB})$

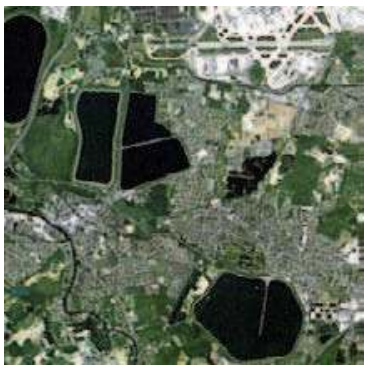

(d) $\hat{\mathbf{z}}$ using (a)

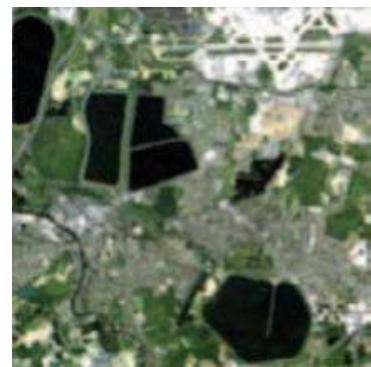

(b) $\mathbf{x}(35 \mathrm{~dB})$

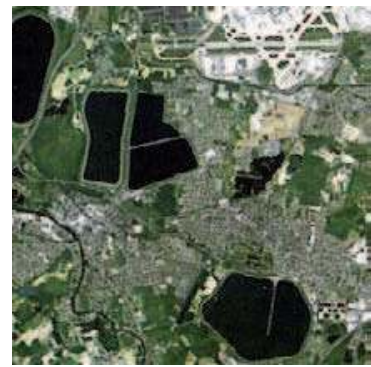

(e) $\hat{\mathbf{z}}$ using (b)

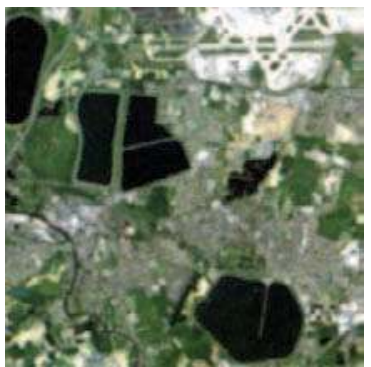

(c) $\mathbf{x}(30 \mathrm{~dB})$

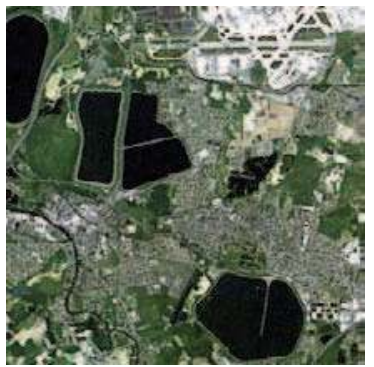

(f) $\hat{\mathbf{z}}$ using (c)

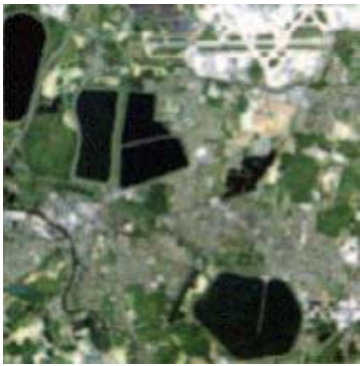

(g) $\mathbf{x}(25 \mathrm{~dB})$

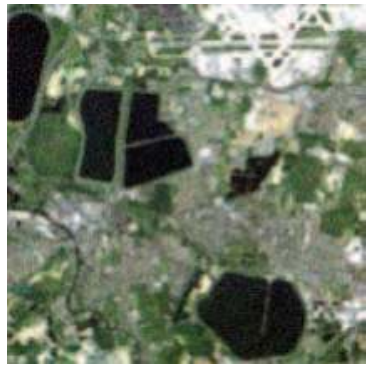

(h) $\mathbf{x}(20 \mathrm{~dB})$

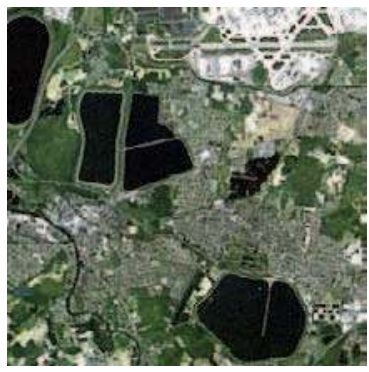

(i) $\hat{\mathbf{z}}$ using (g)

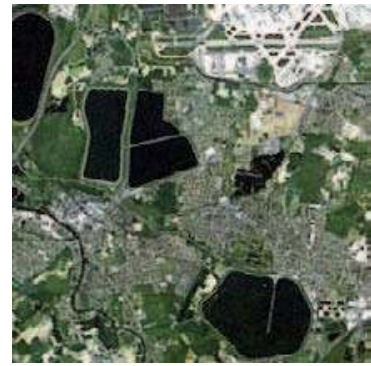

(j) $\hat{\mathbf{z}}$ using (h)

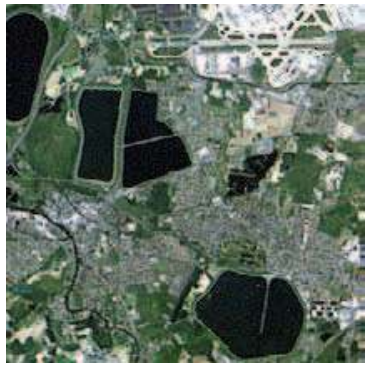

(k) Reference

Fig. 2. Experimental results in Test 2 using $\mathbf{x}$ with different noise levels. 


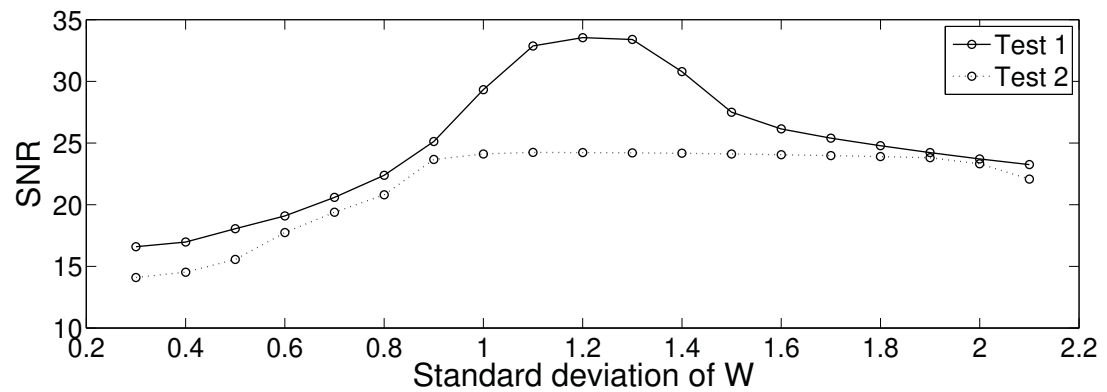

Fig. 3. Influence of $\mathbf{W}$ on fusion performance.

especially in Test 2 (dashed). It can be concluded that for the proposed fusion approach, the exact knowledge about $\mathbf{W}$ is not strictly required. By using a good approximation or estimation of $\mathbf{W}$, fused results with fairly good quality can still be obtained.

In certain practical circumstances, ground truth (reference) may not be available as well, which means there is no prior knowledge about $\mathbf{W}$ at all. The proposed technique can still be applied, by applying Gaussian low-pass filters with increasing $\sigma_{1}$ as $\mathbf{W}$ and validating the results by users' observation. Some fused images from the pansharpening test with different underestimated as well as overestimated $\mathbf{W}$ are shown in Fig. 4.

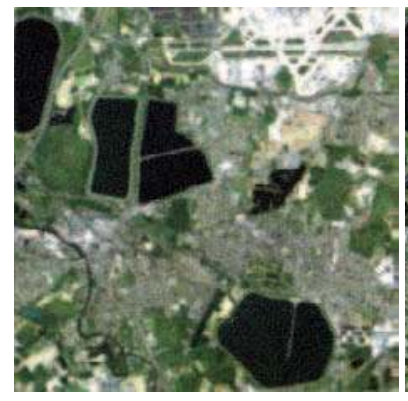

(a) $\sigma_{1}=0.5$

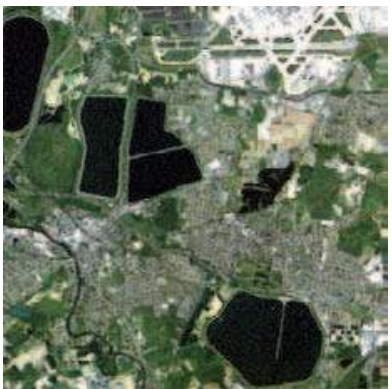

(b) $\sigma_{1}=0.8$

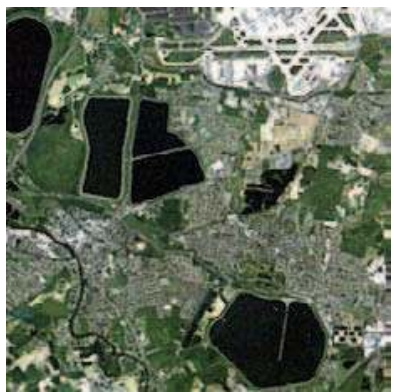

(c) $\sigma_{1}=1.2$

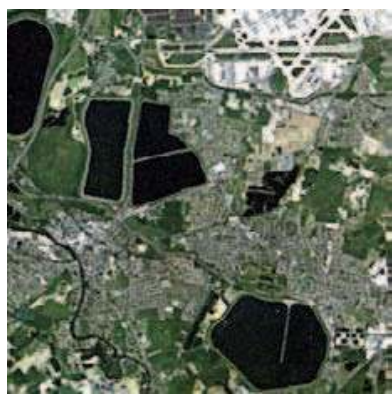

(d) $\sigma_{1}=1.7$

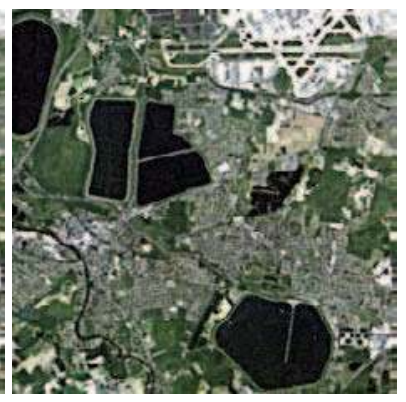

(e) $\sigma_{1}=2.0$

Fig. 4. Fused images using $\mathbf{W}$ with different $\sigma_{1}$. 


\subsubsection{Performance comparison}

In this set of experiments we compare the proposed fusion technique with the EM-based restoration approach of (Duijster et al., 2009) as presented in Section 2.2.1 (denoted as EM-Res), and the Bayesian fusion approach of (Hardie et al., 2004) as presented in Section 2.2.2 (denoted as Bayes-F). To make a fair comparison, all three approaches employ the same statistical parameter estimation strategy.

When performing EM-Res, $\mathbf{W}$ denotes the imaging PSF and reflects the resolution difference between $\mathbf{x}$ and $\mathbf{z}$. Since no high-spatial resolution auxiliary information is utilized, its performance in spatial enhancement is usually quite limited. While in Bayes-F, $\mathbf{W}$ actually reflects the resolution difference between $\mathbf{x}$ and $\mathbf{y}$. Hence, the spatial resolution of $\mathbf{z}$ is limited to that of $\mathbf{y}$. It is notable that both resolution differences may be quite different in practice. However, either of the above two approaches only accounts for one of the resolution differences. The newly proposed fusion approach overcome this limitation, in which $\mathbf{W}$ describes the spatial resolution difference between $\mathbf{x}$ and $\mathbf{z}$, while the resolution difference between $\mathbf{x}$ and $\mathbf{y}$ is accounted for in the covariance estimation. In fact, it combines the advantages of the fusion and restoration techniques, obtaining a result which is actually a weighted result between the results produced by these two techniques. Depending on the spatial resolution differences and noise level in the observation model, it is capable of updating the weights adaptively. If the resolution difference between $\mathbf{x}$ and $\mathbf{y}$ is high, fusion is expected to contribute more to the result than restoration, while if it is low, the restoration part is expected to contribute more.

As a reminder, $\mathbf{W}$ is assumed to be known, but as shown in the first set of experiments, a fair estimation is sufficient. In the following experiments, we have used the knowledge of $\mathbf{W}$. The spatial resolution of $\mathbf{y}$ and thus knowledge about the spatial resolution difference between $\mathbf{x}$ and $\mathbf{y}$ is not required a priori and is estimated during the process.

In order to investigate the performance of different techniques, the following experiment is conducted. Gaussian low-pass filters $\mathbf{G}$ with standard deviation $\sigma_{2} \in[0.3,2.1]$ (with a step of 0.1 ) are applied to the original high-spatial low-spectral image, to generate $\mathbf{y}$ at different spatial resolution scales.

In Fig. 5, the SNRs as a function of $\sigma_{2}$ are shown, the original SNR of $\mathbf{x}$ and the reference is also depicted. The result produced by EM-Res is of course constant since it does not make use of $\mathbf{y}$, while the performance of Bayes-F decreases sharply with decreasing spatial resolution of $\mathbf{y}$ (increase of $\left.\sigma_{2}\right)$. For high spatial resolution of $\mathbf{y}\left(\sigma_{2} \in[0.3,0.9]\right)$, Bayes-F performs better than EM-Res. For higher values of $\sigma_{2}$, which implies the spatial resolution of $\mathbf{y}$ is only slightly higher or even lower than that of $\mathbf{x}$, EM-Res performs better than Bayes-F. When $\sigma_{2}=1.2$, the SNR of the result produced by Bayes-F is almost the same as the original SNR, which well explains the fact that no improvement can be expected by fusing two observations at the same spatial resolution scale. The slightly higher SNR over the original one can be attributed to the noise-resistance of Bayes-F. When the spatial resolution of $\mathbf{y}$ decreases further, Bayes-F does not make a contribution any more, it even deteriorates the $\mathbf{x}$ observation.

As for the proposed approach, three different regimes appear in Fig. 5. For low values of $\sigma_{2}\left(\sigma_{2} \in[0.3,0.6]\right)$, the result of the proposed technique is comparable to the Bayes-F result. For high values of $\sigma_{2}\left(\sigma_{2} \in[1.3,2.1]\right)$, restoration dominates the process and the result of the proposed technique seems to saturate to a value nearby the EM-Res result. This is more obvious in Test 1 , in which general HS and MS image fusion is performed. The middle regime $\left(\sigma_{2} \in[0.6,1.3]\right)$ is the most interesting one. In that regime, both restoration and fusion contribute to the result, leading to an improved fusion performance. 


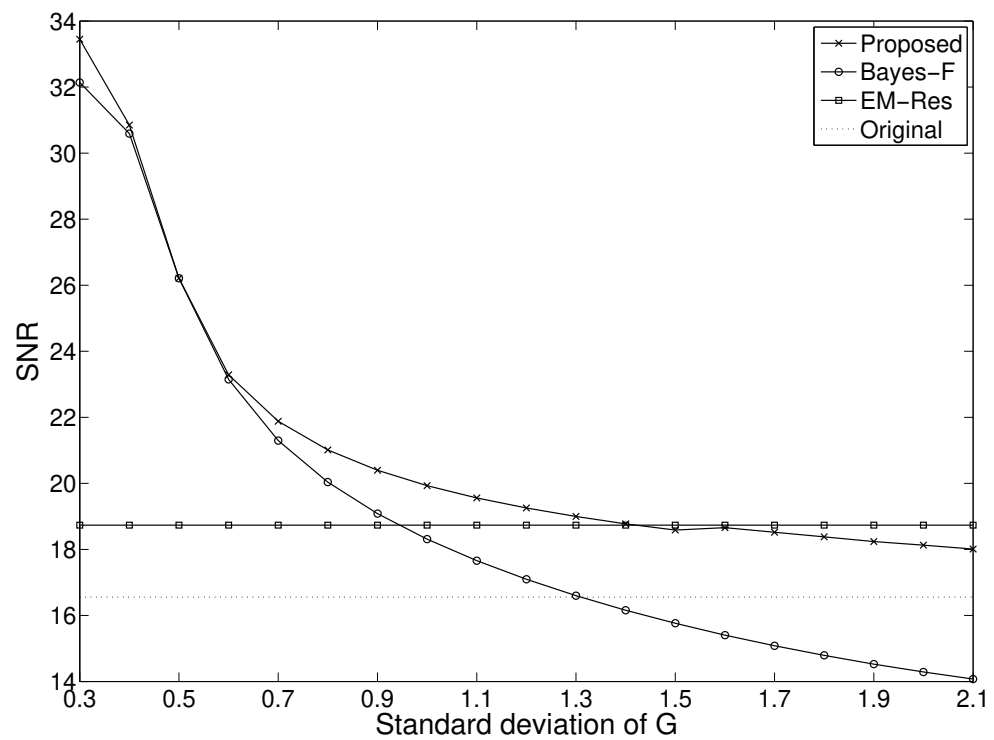

(a) Test 1

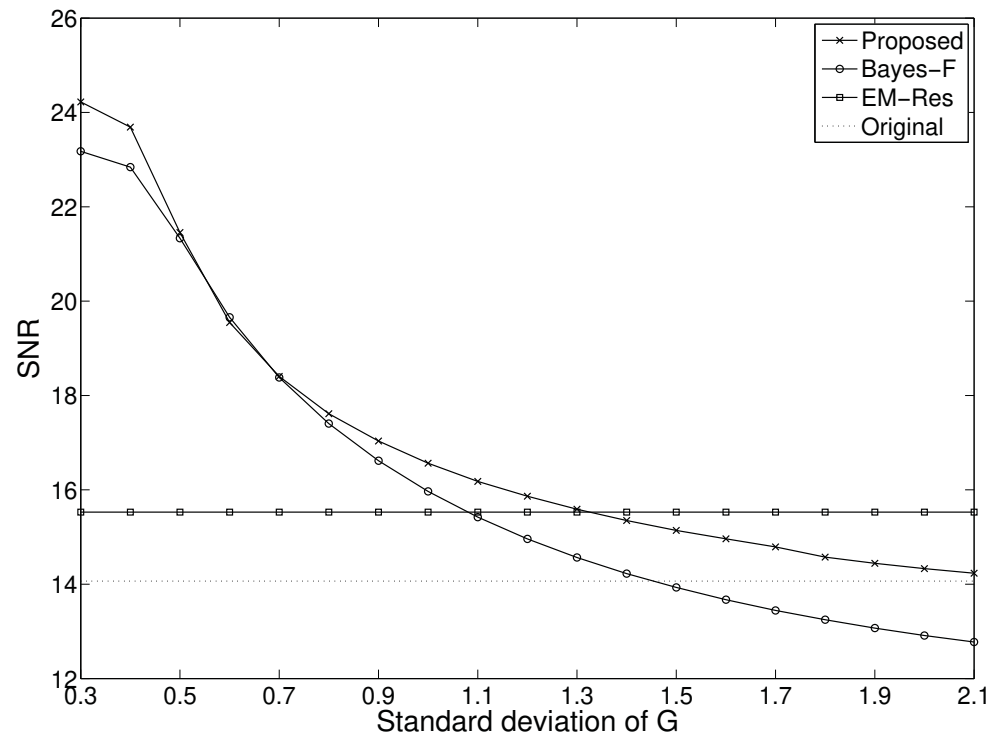

(b) Test 2

Fig. 5. Influence of the spatial resolution of $\mathbf{y}$ on performance. 


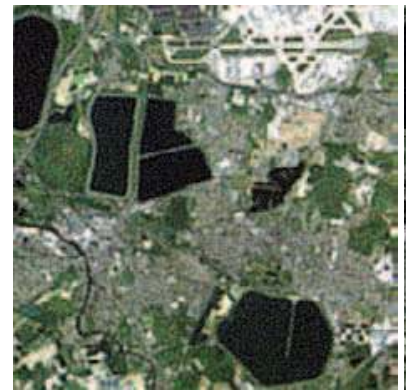

(a) EM-Res

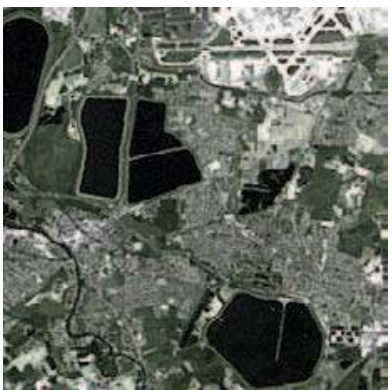

(b) Bayes-F $\left(\sigma_{2}=0\right)$

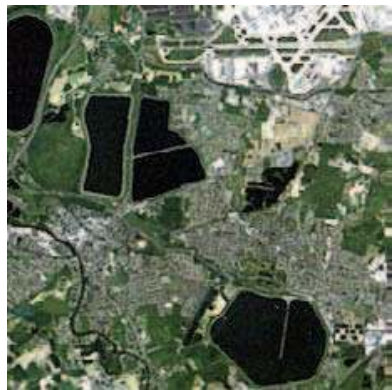

(c) Proposed $\left(\sigma_{2}=0\right)$

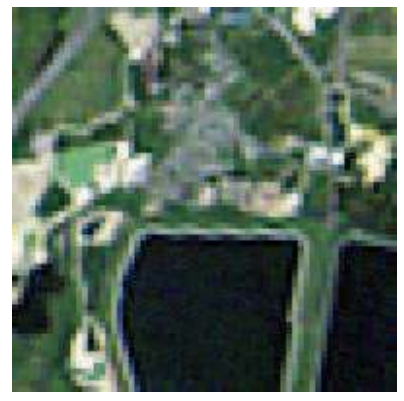

(d) Zoomed reference

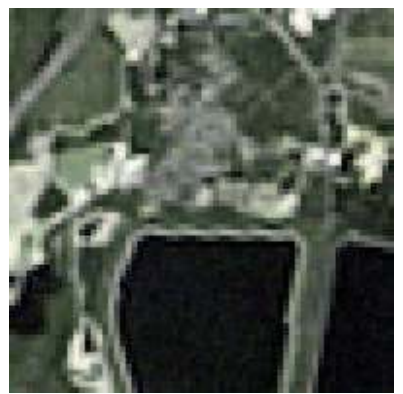

(f) Zoomed (b)

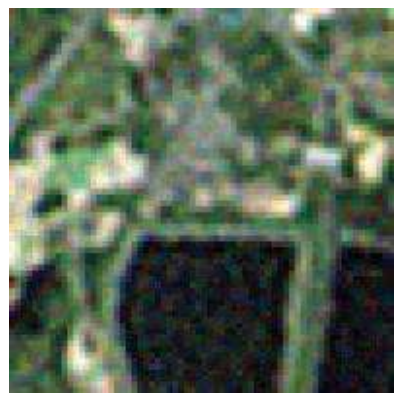

(e) Zoomed (a)

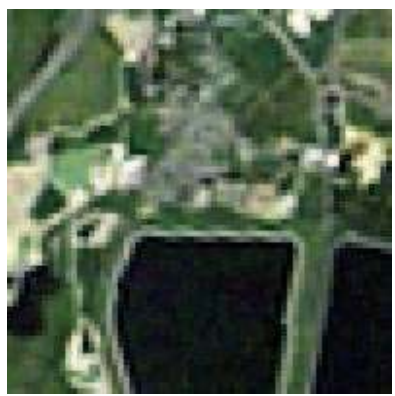

(g) Zoomed (c)

Fig. 6. Experimental results from Test 2.

In Fig. 6, the experimental results of Test 2 are depicted, produced by EM-Res, Bayes-F and the proposed approach. Zoomed images of fusion results produced by Bayes-F and the proposed approach using $\mathbf{y}$ on different spatial resolution scales are also depicted in Fig. 7. It can be observed that for EM-Res, the spatial resolution improvement is limited and the result is quite noisy. The spatial resolution improvements of the results produced by Bayes-F and the proposed approach are comparable. When $\mathbf{y}$ is of lower spatial resolution, the spatial resolution improvement of the proposed approach is better than that of Bayes-F. However, severe spectral distortion (color difference with the reference) can be observed in Bayes-F result. 


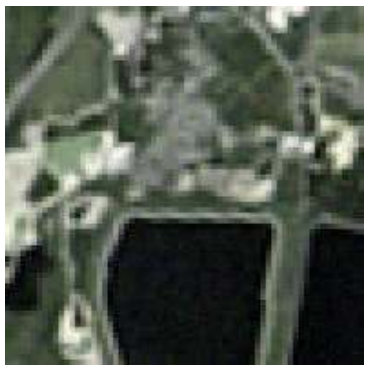

(a) Bayes-F ( $\left.\sigma_{2}=0.5\right)$

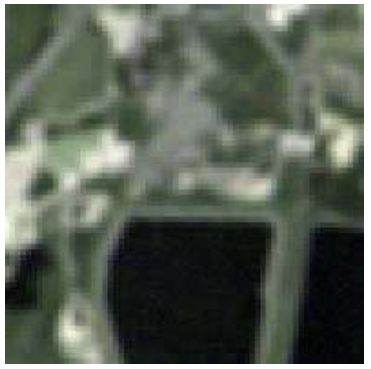

(c) Bayes-F $\left(\sigma_{2}=1.0\right)$

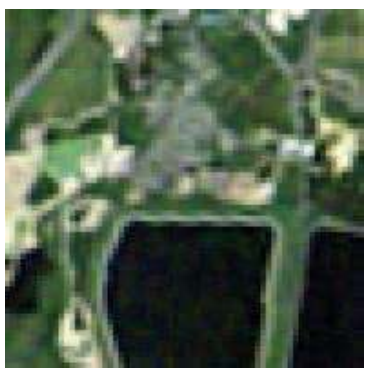

(e) Proposed $\left(\sigma_{2}=0.5\right)$

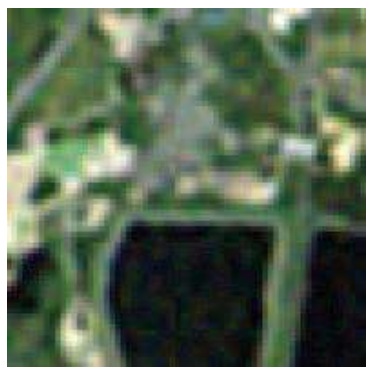

(g) Proposed $\left(\sigma_{2}=1.0\right)$

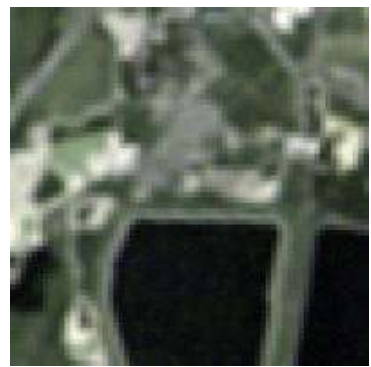

(b) Bayes-F $\left(\sigma_{2}=0.8\right)$

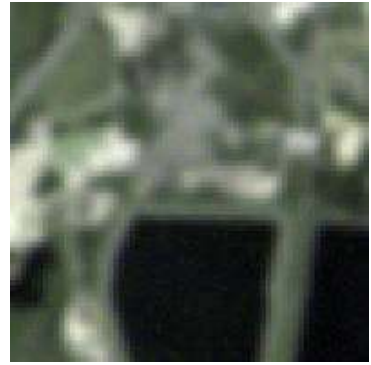

(d) Bayes-F ( $\left.\sigma_{2}=1.3\right)$

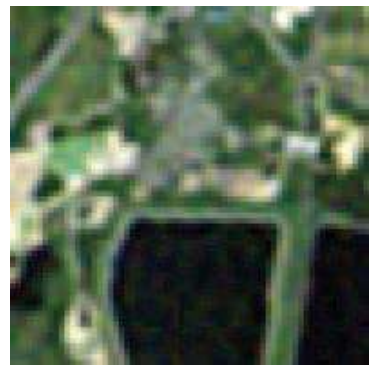

(f) Proposed $\left(\sigma_{2}=0.8\right)$

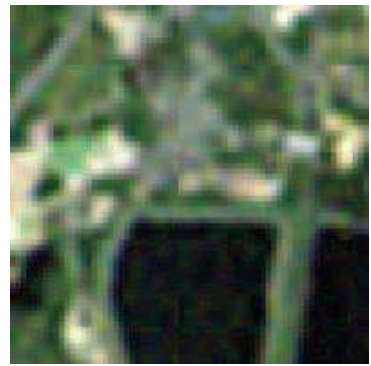

(h) Proposed $\left(\sigma_{2}=1.3\right)$

Fig. 7. Zoomed results in Test 2. 
These findings reflect some weaknesses of EM-Res and Bayes-F approaches. EM-Res approach combines in each iteration a deconvolution step and a denoising step, the latter being a regularization step. It is known that such iterative processes sometimes overemphasize details and thereby tend to amplify the noise. On the other hand, the jeopardy with Bayes-F approach is that it may lose spectral fidelity of the low-spatial high-spectral resolution image by including spatial details from the high-spatial low-spectral resolution image. The proposed approach seems to be able to control both aspects by weighting of the contributions of restoration and fusion.

\section{Conclusion}

In this paper, a fusion approach for two observations (a low-spatial high-spectral resolution observation $\mathbf{x}$ and a high-spatial low-spectral resolution observation $\mathbf{y}$ ) is proposed. The newly proposed fusion approach employs an iterative EM algorithm as well as a Bayesian fusion scheme, in which an image restoration process for $\mathbf{x}$ is applied in combination with a fusion of $\mathbf{x}$ and $\mathbf{y}$. In the simulation experiments, the proposed approach is validated and analyzed, as well as compared with some state-of-the-art techniques which clearly illustrates its advantages.

\section{References}

Aiazzi, B., Alparone, L., Baronti, S. \& Garzelli, A. (2002). Context-driven fusion of high spatial and spectral resolution images based on oversampled multiresolution analysis, IEEE Transaction on Geoscience and Remote Sensing 40(10): 2300-2312.

Alparone, L., Baronti, S., Garzelli, A. \& Nencini, F. (2004). Landsat ETM+ and SAR image fusion based on generalized intensity modulation, IEEE Transaction on Geoscience and Remote Sensing 42(12): 2832-2839.

Alparone, L., Wald, L., Chanussot, J., Thomas, C., Gamba, P. \& Bruce, L. (2007). Comparison of pansharpening algorithms: outcome of the 2006 GRS-S data-fusion contest, IEEE Transaction on Geoscience and Remote Sensing 45(10): 3012-3021.

Carper, W. J., Lillesand, T. M. \& Kiefer, R. W. (1990). The use of Intensity-Hue-Saturation transform for merging SPOT panchromatic and multispectral image data, Photogrammetric Engineering and Remote Sensing 56(4): 459-467.

Chang, C.-I. (2003). Hyperspectral imaging: techniques for spectral detection and classification, Kluwer Academic Publishers.

Chavez, P. S., Stuart, J., Sides, C. \& Anderson, J. A. (1991). Comparison of three different methods to merge multiresolution and multispectral data: Landsat TM and SPOT panchromatic, Photogrammetric Engineering and Remote Sensing 57: 295-303.

Donoho, D. \& Johnstone, I. (1995). Adapting to unknown smoothness via wavelet shrinking, Journal of the American Statistical Association 90(432): 1200-1224.

Duijster, A., Scheunders, P. \& De Backer, S. (2009). Wavelet-based EM algorithm for multispectral-image restoration, IEEE Transaction on Geoscience and Remote Sensing 47(11): 3892-3898.

Edwards, K. \& Davis, P. A. (1994). The use of Intensity-Hue-Saturation transform for producing color shaded-relief images, Photogrammetric Engineering and Remote Sensing 60(11): 1369-1374.

Eismann, M. T. \& Hardie, R. C. (2004). Application of the stochastic mixing model to hyperspectral resolution enhancement, IEEE Transaction on Geoscience and Remote 
Sensing 42(9): 1924-1933.

Eismann, M. T. \& Hardie, R. C. (2005). Hyperspectral resolution enhancement using high-resolution multispectral imagery with arbitrary response functions, IEEE Transaction on Geoscience and Remote Sensing 43(3): 455-465.

Figueiredo, M. A. T. \& Nowak, R. D. (2003). An EM algorithm for wavelet-based image restoration, IEEE Transaction on Image Processing 12(8): 906-916.

Gomez, R., Jazaeri, A. \& Kafatos, M. (2001). Wavelet-based hyperspectral and multi-spectral image fusion, Proceedings of SPIE 4383: 36-42.

Hardie, R. C., Eismann, M. T. \& Wilson, G. L. (2004). MAP estimation for hyperspectral image resolution enhancement using an auxiliary sensor, IEEE Transaction on Image Processing 13(9): 1174-1184.

Liu, J. G. \& Moore, J. M. (1998). Pixels block intesnsity modulation: adding spatial detail to TM band 6 thermal imagery, International Journal of Remote Sensing 19(13): 2477-2491.

Núñez, J., Otazu, X., Fors, O., Prades, A., Palà, V. \& Arbiol, R. (1999). Multiresolution-based image fusion with additive wavelet decomposition, IEEE Transaction on Geoscience and Remote Sensing 37(3): 1204-1211.

Pohl, C. \& Van Genderen, J. L. (1998). Multi-sensor image fusion in remote sensing: concepts, methods and applications, International Journal of Remote Sensing 19(5): 823-854.

Shettigara, V. K. (1992). A generalized component substitution technique for spatial enhancement of multispectral images using a higher resolution data set, Photogrammetric Engineering and Remote Sensing 58: 561-567.

Shi, W. Z., Zhu, C. Q., Zhu, C. Y. \& Yang, X. M. (2003). Multi-band wavelet for fusing SPOT panchromatic and multispectral images, Photogrammetric Engineering and Remote Sensing 69(5): 513-520.

Tu, T. M., Huang, P. S., Hung, C. L. \& Chang, C. P. (2004). A fast intensity-hue-saturation fusion technique with spectral adjustment for IKONOS imagery, IEEE Geoscience and Remote Sensing Letters 1(4): 309-312.

Tu, T. M., Su, S. C., Shyu, H. C. \& Huang, P. S. (2001). A new look at IHS-like image fusion methods, Information Fusion 2(3): 177-186.

Wang, Z., Ziou, D., Armenakis, C., Li, D. \& Li, Q. (2005). A comparative analysis of image fusion methods, IEEE Transaction on Geoscience and Remote Sensing 43(6): 1391-1402.

Zhang, Y. \& He, M. (2007). Multi-spectral and hyperspectral image fusion using 3-D wavelet transform, Journal of Electronics(China) 24(2): 218-224. 


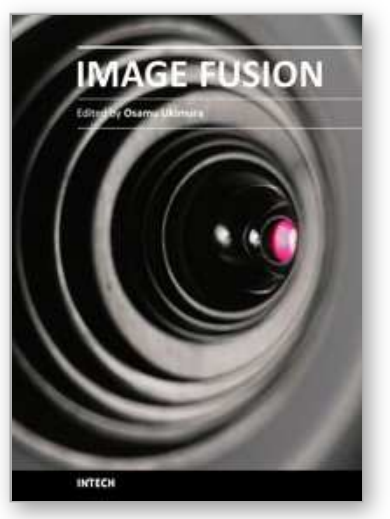

\author{
Image Fusion \\ Edited by Osamu Ukimura
}

ISBN 978-953-307-679-9

Hard cover, 428 pages

Publisher InTech

Published online 12, January, 2011

Published in print edition January, 2011

Image fusion technology has successfully contributed to various fields such as medical diagnosis and navigation, surveillance systems, remote sensing, digital cameras, military applications, computer vision, etc. Image fusion aims to generate a fused single image which contains more precise reliable visualization of the objects than any source image of them. This book presents various recent advances in research and development in the field of image fusion. It has been created through the diligence and creativity of some of the most accomplished experts in various fields.

\title{
How to reference
}

In order to correctly reference this scholarly work, feel free to copy and paste the following:

Yifan Zhang (2011). EM-Based Bayesian Fusion of Hyperspectral and Multispectral Images, Image Fusion, Osamu Ukimura (Ed.), ISBN: 978-953-307-679-9, InTech, Available from:

http://www.intechopen.com/books/image-fusion/em-based-bayesian-fusion-of-hyperspectral-and-multispectralimages

\section{INTECH}

open science / open minds

\section{InTech Europe}

University Campus STeP Ri

Slavka Krautzeka 83/A

51000 Rijeka, Croatia

Phone: +385 (51) 770447

Fax: +385 (51) 686166

www.intechopen.com

\section{InTech China}

Unit 405, Office Block, Hotel Equatorial Shanghai

No.65, Yan An Road (West), Shanghai, 200040, China

中国上海市延安西路65号上海国际贵都大饭店办公楼 405 单元

Phone: +86-21-62489820

Fax: +86-21-62489821 
(C) 2011 The Author(s). Licensee IntechOpen. This chapter is distributed under the terms of the Creative Commons Attribution-NonCommercialShareAlike-3.0 License, which permits use, distribution and reproduction for non-commercial purposes, provided the original is properly cited and derivative works building on this content are distributed under the same license. 